K. AZUKAWA

KODAI MATH. J.

14 (1991), 1-12

\title{
A NOTE ON CARATHÉODORY AND KOBAYASHI PSEUDODISTANCES
}

\author{
By Kazuo Azukawa
}

\section{Introduction.}

Recently, Jarnicki and Pflug [7], [8] presented an effective formula for the Carathéodory pseudodistance from the origin on logarithmically coned, complete Reinhardt domains in $\boldsymbol{C}^{n}$. The aim of this note is to establish the wasteless formula for the pseudodistance from the origin on such domains (Theorem 2.2). We also apply this formula to the case of dimension two and represent the pseudodistance by means of the continued fraction expansion of real numbers (Theorem 4.1).

\section{Preliminary.}

Let $D$ be a domain in $C^{n}$. For $p, q \in D$, let

$$
\begin{gathered}
c_{D}^{*}(p, q)=\sup \{|f(q)| ; f \in \operatorname{Hol}(D, U), f(p)=0\}, \\
k_{D}^{*}(p, q)=\inf \{t ; 0 \leqq t<1, \text { there exists an } f \in \operatorname{Hol}(U, D) \\
\text { such that } f(0)=p \text { and } f(t)=q\},
\end{gathered}
$$

and

$g_{D}(p, q)=\sup \{f(q) ; f$ is a negative plurisubharmonic function on $D$ such that $\left.\limsup _{z \rightarrow p}(f(z)-\log |z-p|)<+\infty\right\}$,

where $U$ is the unit disc in $C$ and, for complex manifolds $X$ and $Y, \operatorname{Hol}(X, Y)$ denotes the set of all holomorphic mappings from $X$ into $Y$. The function $c_{D}=\tanh ^{-1} c_{D}^{*}$ (resp. the largest pseudodistance $k_{D}$ on $D$ dominated by $k_{D}^{1}:=$ $\tanh ^{-1} k_{D}^{*}$ ) is called the Carathéodory (resp. Kobayashi) pseudodistance on $D$, and the function $g_{D}(p, \cdot)$ is called the pluri-complex Green function on $D$ with pole at $p$ (cf., e.g., [2], [3], [4], [5], [10], [11], [15]). These functions $c_{D}^{*}, k_{D}^{*}$, and $g_{D}$ have the decreasing property for holomorphic mappings and satisfy

$$
c_{D}^{*} \leqq \exp g_{D} \leqq k_{D}^{*} \quad \text { on } D \times D
$$

(see $[10])$.

Received February 13, 1990. 
We shall show the following lemma, part (ii) of which is well-known ([7], [8]).

LEMMA 1.1. Let $D$ be a balanced domain in $\boldsymbol{C}^{n}$ with the Minkowski functional $\mu_{D}(z)=\inf \{r>0 ; z \in r D\}$, where $r D=\{r z ; z \in D\}$.

(i) $\quad c_{D}^{*}(0, \cdot) \leqq \exp g_{D}(0, \cdot) \leqq k *(0, \cdot) \leqq \mu_{D}$ on $D$.

(ii) $D$ is convex if and only if $c_{D}^{*}(0, \cdot)=\mu_{D}$ on $D$.

(iii) $D$ is pseudoconvex if and only if $\exp g_{D}(0, \cdot)=\mu_{D}$ on $D$.

Proof. (i) In view of (1.1) we have to prove only the last inequality. Let $z \in D=\left\{z \in C^{n} ; \mu_{D}(z)<1\right\}$, and take $r$ with $\mu_{D}(z)<r<1$. Then the function $f(\lambda)$ $=\lambda z / r, \lambda \in U$ belongs to $\operatorname{Hol}(U, D)$ and satisfies $f(r)=z$, so that $k_{D}^{*}(0, z) \leqq r$. Thus, $k_{D}^{*}(0, z) \leqq \mu_{D}(z)$. (iii) Assume $\exp g_{D}(0, \cdot)=\mu_{D}$ on $D$. Then, $\log \mu_{D}=$ $g_{D}(0, \cdot)$ is plurisubharmonic on $D$, and so on $\boldsymbol{C}^{n}$. Hence, the balanced domain $D$ is pseudoconvex. Conversely assume that $D$ is pseudoconvex. Then, $\log \mu_{D}$ is plurisubharmonic on $D([1])$. Since $\mu_{D}$ is continuous at 0 it follows that limsup $_{z \rightarrow 0}\left(\log \mu_{D}(z)-\log |z|\right)<+\infty$, so that $\log \mu_{D} \leqq g_{D}(0, \cdot)$. The proof is completed.

As an application of Lemma 1.1 we obtain Kubota's theorem on symmetric bounded domains.

COROLlary 1.2 (Kubota [13], [12]). Let $D$ be a symmetric bounded domain in $\boldsymbol{C}^{n}$ realized as a convex balanced domain. Then,

$$
\begin{array}{r}
c_{D}^{*}(z, w)=\inf \{r ; 0<r<1, \text { there exists an } F \in \operatorname{Aut}(D) \\
\text { such that } F(w)=0 \text { and } F(z) \in r D\}
\end{array}
$$

for all $z, w \in D$, and

$$
\left\{z \in D ; c_{D}^{*}(0, z)<r\right\}=r D
$$

for all $r>0$ with $r<1$, where Aut $(D)$ is the set of all holomorphic automorphisms of $D$.

Proof. To prove the formula (1.2) we denote by $d_{w}(z)$ the right hand side of (1.2). Since $D$ is homogeneous, $K_{w}:=\{F \in \operatorname{Aut}(D) ; F(w)=0\}$ is not empty. It follows that

$$
\begin{aligned}
d_{w}(z) & =\inf \bigcup_{F \in K_{w}}\{r ; 0<r<1, F(z) \in r D\} \\
& =\inf _{F \in K_{w}} \inf \{r ; 0<r<1, F(z) \in r D\} \\
& =\inf _{F \in K_{w}} \mu_{D}(F(z)) .
\end{aligned}
$$

Since $D$ is convex, by Lemma 1.1 (ii) and the biholomorphic invariance of $c_{D}^{*}$ we see that for every $F \in K_{w}, \mu_{D}(F(z))=c_{D}^{*}(0, F(z))=c_{D}^{*}(w, z)$; therefore, $\mu_{D}(F(z))$ does not depend on the choice of $F \in K_{w}$ and (1.2) is established. The relation 
(1.3) is a direct consequence of the equality $\mu_{D}=c_{D}^{*}(0, \cdot)$. The proof is completed.

Under the hypothesis in Corollary 1.2 it is well-known ([11], [14]) that $c_{D}=k_{D}$. Using only the homogeneity and the convex balancedness of $D$, we can show this as follows: By Lemma 1.1 (i) and (ii) we have $c_{D}^{*}(0, \cdot)=k_{D}^{*}(0, \cdot)$. From the homogeneity of $D$ and the biholomorphic invariance of $c_{D}^{*}$ and $k_{D}^{*}$ it follows that $c_{D}^{*}=k_{D}^{*}$; therefore, $c_{D}=k_{D}^{1}$ and $k_{D}^{1}$ is a pseudodistance, so that $c_{D}=$ $k_{D}^{1}=k_{D}$.

\section{Logarithmically coned Reinhardt domains.}

Let $D$ be a Reinhardt domain in $\boldsymbol{C}^{n}$ with the real representative domain $|D|=\left\{\left(\left|z_{1}\right|, \cdots,\left|z_{n}\right|\right) \in\left(\boldsymbol{R}_{+}\right)^{n} ;\left(z_{1}, \cdots, z_{n}\right) \in D\right\}$, where $\boldsymbol{R}_{+}=\{x \in R ; x \geqq 0\}$. Assume $D$ is $\operatorname{logarithmically~coned,~that~is,~the~set~} \log |D|:=\left\{x=\left(x_{1}, \cdots, x_{n}\right) \in \boldsymbol{R}^{n} ; e^{x}:=\right.$ $\left.\left(e^{x_{1}}, \cdots, e^{x_{n}}\right) \in|D|\right\}$ is a cone in $\boldsymbol{R}^{n}$ with vertex at the origin. Set $\boldsymbol{Z}_{+}=\boldsymbol{Z} \cap \boldsymbol{R}_{+}$ and, for a subset $S$ of $\boldsymbol{R}^{n}$, set $S_{*}=S \backslash\{0\}$. For $z=\left(z_{1}, \cdots, z_{n}\right) \in \boldsymbol{C}^{n}$ and $\alpha=$ $\left(\alpha_{1}, \cdots, \alpha_{n}\right) \in\left(\boldsymbol{Z}_{+}\right)^{n}$, set $z^{\alpha}=z_{1}^{\alpha_{1}} \cdots z_{n}{ }^{\alpha}$. Let $S_{D}=\left\{\alpha \in\left(\boldsymbol{Z}_{+}\right)_{*}^{n} ;\left|z^{\alpha}\right|<1\right.$ for all $\left.z \in D\right\}$, and let $S_{D}+S_{D}=\left\{\alpha+\beta ; \alpha, \beta \in S_{D}\right\}$. A typical example of logarithmically coned, complete Reinhardt domains is $\left\{z \in C^{n} ;\left|z^{\alpha}\right|<1\right.$ for all $\left.\alpha \in T\right\}$, where $T$ is a finite subset of $\left(\boldsymbol{Z}_{+}\right)_{*}^{n}$.

Jarnicki and Pflug proved the following.

LEMma 2.1 ([7; Theorem 2], [8; Theorem 2.1]). Let $D$ be a logarithmically coned, complete Reinhardt domain in $\boldsymbol{C}^{n}$ with $S_{D}$. It then holds that for $z \in D$,

$$
\begin{aligned}
c_{D}^{*}(0, z) & =\sup \left\{\left|z^{\alpha}\right| ; \alpha \in S_{D}\right\} \\
& =\sup \left\{\left|z^{\alpha}\right| ; \alpha \in S_{D} \backslash\left(S_{D}+S_{D}\right)\right\} .
\end{aligned}
$$

The aim of this section is to prove a precision of Lemma 2.1.

Let $C$ be a closed subset of $\boldsymbol{R}^{n}$. A point $x$ in $C$ is called a vertex of $C$ if there exists a linear functional $f$ on $\boldsymbol{R}^{n}$ and a number $c \in \boldsymbol{R}$ such that $f(x)=c$ and $f(y)<c$ for all $y \in C \backslash\{x\}$, that is, $x$ is a vertex of the convex hull Conv $C$ of $C$ in the usual sense. By Vert $C$ we denote the set of all vertices of $C$.

Our result is the following.

THEOREM 2.2. Let $D$ be a logarithmically coned, complete Reinhardt domain in $\boldsymbol{C}^{n}$ with $S_{D}$. Then,

$$
c_{D}^{*}(0, z)=\max \left\{\left|z^{\alpha}\right| ; \alpha \in \operatorname{Vert} S_{D}\right\}
$$

for $z \in D$.

It follows from the definition that

$$
S_{D}=\left\{\alpha \in\left(\boldsymbol{Z}_{+}\right)_{*}^{n} ;\langle\alpha, y\rangle<0 \text { for all } y \in \log |D|\right\} .
$$

To prove Theorem 2.2 , by virtue of Lemma 2.1 we must show that if 


$$
\Phi(x)=\sup \left\{\langle x, \alpha\rangle ; \alpha \in S_{D}\right\}
$$

for $x \in \log |D|$, then

$$
\Phi(x)=\max \left\{\langle x, \alpha\rangle ; \alpha \in \operatorname{Vert} S_{D}\right\} .
$$

We need a lemma.

LEMmA 2.3. Let $x \in \log |D|$. Let $\left(x_{j}\right)_{j}$ be a sequence in $\boldsymbol{R}^{n}$ and $\left(\beta_{j}\right)_{j}$ be a sequence in $S_{D}$ such that $x_{j} \rightarrow x$ as $j \rightarrow \infty$ and the sequence $\left(\left\langle x_{j}, \beta_{j}\right\rangle\right)_{j}$ is bounded. Then, $\left(\beta_{\jmath}\right)$ is bounded.

Proof. Suppose $\left(\beta_{j}\right)$ is not bounded. We may assume that $\left|\beta_{j}\right| \rightarrow+\infty$ and $\beta_{j} /\left|\beta_{j}\right| \rightarrow \xi$ as $j \rightarrow \infty$ for some $\xi \in \boldsymbol{R}^{n}$ with $|\xi|=1$. Since $\left(\left\langle x_{j}, \beta_{j}\right\rangle\right)$ is bounded, it follows that $\langle x, \xi\rangle=\lim _{\jmath \rightarrow \infty}\left\langle x, \beta_{\jmath}\right\rangle /\left|\beta_{j}\right|=0$. Take an $\varepsilon>0$ so that $x+\varepsilon \xi \in$ $\log |D|$. We have

$$
\lim _{\jmath \rightarrow \infty}\left\langle x+\varepsilon \xi, \beta_{\jmath}\right\rangle=\lim _{\jmath \rightarrow \infty}\left\langle x+\varepsilon \xi, \beta_{j} / \beta_{j} \mid\right\rangle \cdot \lim _{\jmath \rightarrow \infty}\left|\beta_{j}\right|=+\infty ;
$$

therefore $\left\langle x+\varepsilon \xi, \beta_{\jmath}\right\rangle>0$ for some $j$. This contradicts the facts $x+\varepsilon \xi \in \log |D|$ and $\beta_{j} \in S_{D}$, and completes the proof.

Proof of Theorem 2.2. To prove (2.3) fix any $x \in \log |D|$. We first note that

$$
\Phi(x)=\max \left\{\langle x, \alpha\rangle ; \alpha \in S_{D}\right\} .
$$

Indeed, let $\left(\alpha_{\jmath}\right)$ be a sequence in $S_{D}$ such that $\Phi(x)=\lim _{\jmath \rightarrow \infty}\left\langle x, \alpha_{\jmath}\right\rangle$. By Lemma 2.3, $\left(\alpha_{\jmath}\right)$ is bounded, so that we may assume that $\left(\alpha_{\jmath}\right)$ converges to a point $\alpha \in S_{D}$; therefore $\Phi(x)=\langle x, \alpha\rangle$. Then $H:=\left\{y \in \boldsymbol{R}^{n} ;\langle x, y\rangle=\Phi(x)\right\}$ is a supporting hyperplane of Conv $S_{D}$. By Lemma 2.3 we see that $S_{D} \cap H$ is bounded to the effect that $S_{D} \cap H$ is a finite set. Let $\alpha \in S_{D} \cap H$ be a vertex of $S_{D} \cap H$ in $H$. We shall show that $\alpha$ is also a vertex of $S_{D}$. Take $\eta \in H_{*}$ and $c \in \boldsymbol{R}$ such that

$$
\langle y, \eta\rangle\left\langle c \text { for all } y \in S_{D} \cap H \backslash\{\alpha\} \text { and }\langle\alpha, \eta\rangle=c .\right.
$$

Take an $\varepsilon_{0}>0$ such that $x+\varepsilon \eta \in \log |D|$ for all $\varepsilon>0$ with $\varepsilon<\varepsilon_{0}$. We note that if $0<\varepsilon<\varepsilon_{0}$, then

$$
\langle x+\varepsilon \eta, \alpha\rangle=\Phi(x)+\varepsilon c .
$$

In view of (2.6), to prove that $\alpha \in \operatorname{Vert} S_{D}$, it is sufficient to show the following:

There exists an $\varepsilon$ such that $0<\varepsilon<\varepsilon_{0}$ and $\langle x+\varepsilon \eta, \beta\rangle\langle\Phi(x)+\varepsilon c$ for all $\beta \in S_{D} \backslash\{\alpha\}$.

Now suppose the statement (2.7) does not hold, and take sequences $\left(\varepsilon_{\jmath}\right)$ and $\left(\alpha_{\jmath}\right)$ such that $0<\varepsilon_{j}<\varepsilon_{0}, \alpha_{j} \in S_{D} \backslash\{\alpha\},\left\langle x+\varepsilon_{j} \eta, \alpha_{j}\right\rangle \geqq \Phi(x)+\varepsilon_{j} c$ for all $j$, and $\lim _{i_{j \rightarrow \infty}} \varepsilon_{j}$ 
$=0$. Since $\left\langle x+\varepsilon_{j} \eta, \alpha_{j}\right\rangle<0$ for all $j$, by Lemma $2.3\left(\alpha_{\jmath}\right)$ is bounded. We may assume that $\alpha_{j} \rightarrow \gamma \in S_{D} \backslash\{\alpha\}$. Then, $\langle x, \gamma\rangle \geqq \Phi(x)$, so that $\gamma \in H$; therefore (2.5) implies that

$$
\langle\eta, \gamma\rangle\langle c .
$$

On the other hand, $\alpha_{j}=\gamma$ for sufficiently large $j$, and $\left\langle x+\varepsilon_{j} \eta, \gamma\right\rangle \geqq \Phi(x)+\varepsilon_{j} c$, or $\langle\eta, \gamma\rangle \geqq c$, which contradicts (2.8). The proof is completed.

\section{Half-regular continued fraction expansions.}

Let $\left(a_{j}\right)_{j \geqq 0}$ be a sequence of integers with

$$
a_{j} \geqq 2 \quad(j \geqq 1) .
$$

Consider the mappings $s_{j}(w)=a_{j}-1 / w$,

We say that

$$
\begin{aligned}
s_{0} \circ \cdots \circ s_{\jmath-1}(w) & =a_{0}-\frac{1}{a_{1}}-\cdots-\frac{1}{a_{\jmath-1}}-\frac{1}{w} \\
& =:\left[a_{0}, a_{1}, \cdots, a_{\jmath-1}, w\right] \quad(j \geqq 1) .
\end{aligned}
$$

$$
\left[a_{0}, a_{1}, \cdots, a_{\jmath-1}\right]=a_{0}-\frac{1}{a_{1}}-\cdots-\frac{1}{a_{\jmath-1}}
$$

is a half-regular continued fraction. For $j \geqq 1$, let $p_{j} / q_{j}=\left[a_{0}, \cdots, a_{\jmath-1}\right]$ be the natural representation, i.e., $p_{j}$ and $q_{j}>0$ are relatively prime integers. For convenience, let $\left(q_{0}, p_{0}\right)=(0,1)$. It is easily seen that $\left(q_{1}, p_{1}\right)=(1,0)$,

$$
\begin{gathered}
\left(\begin{array}{c}
q_{j+1} \\
p_{j+1}
\end{array}\right)=\left(\begin{array}{ll}
q_{\jmath} & q_{\jmath-1} \\
p_{\jmath} & p_{\jmath-1}
\end{array}\right)\left(\begin{array}{c}
a_{\jmath} \\
-1
\end{array}\right) \quad(j \geqq 1), \\
\operatorname{det}\left(\begin{array}{cc}
q_{\jmath} & q_{\jmath-1} \\
p_{\jmath} & p_{\jmath-1}
\end{array}\right)=1 \quad(j \geqq 1), \\
{\left[a_{0}, \cdots, a_{\jmath-1}, w\right]=\frac{p_{j} w-p_{\jmath-1}}{q_{j} w-q_{\jmath-1}} \quad(j \geqq 1)}
\end{gathered}
$$

(cf., e.g., [9], [16]). It follows from (3.3) and (3.4) that

$$
\begin{aligned}
& \text { the function }\left[a_{0}, \cdots, a_{\jmath-1}, x\right] \text { is strictly increasing } \\
& \text { in the interval } 1 \leqq x<+\infty \text { and has the interval } \\
& {\left[a_{0}, \cdots, a_{\jmath-1}-1\right] \leqq y<\left[a_{0}, \cdots, a_{\jmath-1}\right] \text { as its image. }}
\end{aligned}
$$

Let $\omega$ be a real number. If $\omega$ is rational (resp. irrational), then there exists a unique pair of finite (resp. infinite) sequences $\left(a_{j}\right)_{0 \leq j<N}$ and $\left(\omega_{j}\right)_{1 \leqq j<N}$ with $N$ a positive integer (resp. $N=+\infty$ ) such that (3.1) holds, 


$$
\omega_{j}>1, \quad \omega=\left[a_{0}, \cdots, a_{\jmath-1}, \omega_{j}\right] \quad(1 \leqq j<N),
$$

and $\omega_{N-1}=a_{N-1}$ (resp. and there exist infinitely many $j$ with $a_{\jmath} \geqq 3$ ). The argorithm $\omega \mapsto\left(a_{\jmath}\right)_{0 \leqq \jmath<N}$, symbolically written as

$$
\omega=a_{0}+K_{1 \leqq \jmath<N}\left(-1 / a_{\jmath}\right)=\left[a_{0}, a_{1}, \cdots\right],
$$

is called the half-regular continued fraction expansion of $\omega$; for every $j$, the integer $a$, is called the $j$-th partial quotient, and the rational number $\left[a_{0}, \cdots\right.$, $\left.a_{\jmath-1}\right]$ the $j$-th approximant of the expansion. Representing $\left[a_{0}, \cdots, a_{\jmath-1}\right]=p_{j} / q_{\jmath}$ naturally, we see that

$$
p_{j}-q_{j} \omega=\frac{1}{q_{j} \omega_{j}-q_{j-1}}=\frac{1}{\omega_{1} \cdots \omega_{j}}
$$

and $q_{\jmath} \geqq j(j \geqq 1)$. It follows that the sequence $\left(p_{j} / q_{\jmath}\right)_{\text {, }}$ is decreasingly convergent to $\omega$ (cf., e.g., [16]). When $N$ is finite it is convenient to set

$$
a_{N}=+\infty \text {. }
$$

In this and subsequent sections, by $\operatorname{grad}(\alpha, \beta)$ we denote the gradient of the segment determined by two distinct points $\alpha, \beta$ in $R^{2}$.

LEMMA 3.1. Let $\omega$ be a positive real number and $a_{0}+K_{1 \leqq \jmath<N}\left(-1 / a_{\jmath}\right)$ be its half-regular continued fraction expansion with the natural representations $p_{j} / q_{j}=$ $\left[a_{0}, \cdots, a_{\jmath-1}\right](1 \leqq j<N+1)$, as well as $\left(q_{0}, p_{0}\right)=(0,1)$. Let $\alpha=(0,1), \beta=(1, \omega)$, $S=\left(\boldsymbol{Z}^{2}\right)_{*} \cap\left(\boldsymbol{R}_{+} \alpha+\boldsymbol{R}_{+} \beta\right)$, and $\alpha_{\jmath}=\left(q_{\jmath}, p_{\jmath}\right)(0 \leqq j<N+1)$. It then holds that

(see (3.7)).

$$
\text { Vert } S=\left\{\alpha_{0}\right\} \cup\left\{\alpha_{\jmath} ; 1 \leqq j<N+1, a_{\jmath} \neq 2\right\}
$$

Proof. Let $1 \leqq j<N+1$. It follows from (3.3) that the pair $\left\{\alpha_{\jmath-1}, \alpha_{j}\right\}$ generates the lattice $Z^{2}$, so that there is no elements of $\boldsymbol{Z}^{2}$ in the open triangle determined by $\alpha_{\jmath_{-1}}, \alpha_{\jmath}$, and the origin. Furthermore, we see that $g_{\jmath}:=$ $\operatorname{grad}\left(\alpha_{\jmath-1}, \alpha_{\jmath}\right)$ is given by $g_{\jmath}=\left[a_{0}, \cdots, a_{\jmath-1}-1\right]=\left[a_{0}, \cdots, a_{\jmath-1}, 1\right]$ (by (3.4)), so that $g_{j+1} \geqq g_{j}$ and that the equality holds if and only if $a_{\jmath}=2$ (by (3.5)). It is trivial that $\alpha_{0} \in \operatorname{Vert} S$ and that $\alpha_{N} \in \operatorname{Vert} S$ when $N$ is finite. Thus, the assertion follows.

Remark 3.2. Under the assumptions in Lemma 3.1 we see that $S \backslash(S+S)$ $=\left\{\alpha_{\jmath} ; 0 \leqq j<N+1\right\}$.

LEMMA 3.3. Let $a_{\imath}, b_{j} \in \boldsymbol{Z}, a_{0} \geqq 1, b_{0} \geqq 1, a_{\imath} \geqq 2(1 \leqq i \leqq k-1), b_{\jmath} \geqq 2(1 \leqq j \leqq l-1)$. If $\left[a_{0}, \cdots, a_{k-1}\right]=\left[b_{0}, \cdots, b_{l-1}\right]^{-1}$, then

(i) $\left[a_{0}, \cdots, a_{k-1}-1\right]=\left[b_{0}, \cdots, b_{l-2}\right]^{-1}$ provided that $a_{k-1} \geqq 2$,

(ii) $a_{0}+\cdots+a_{k-1}-k=b_{0}+\cdots b_{l-1}-l$, and

(iii) $a_{k-1}=2$ or $b_{l-1}=2$ provided that $a_{k-1} \geqq 2$. 
Proof. We first note that if $p_{j} / q_{j}=\left[a_{0}, \cdots, a_{\jmath-1}\right](j=k-1, k)$ are natural representations, then

$$
\begin{aligned}
& {\left[a_{0}, \cdots, a_{k-1}, 2\right]=\frac{p_{k}+\left(p_{k}-p_{k-1}\right)}{q_{k}+\left(q_{k}-q_{k-1}\right)},} \\
& {\left[a_{0}, \cdots, a_{k-1}+1\right]=\frac{p_{k}+p_{k-1}}{q_{k}+q_{k-1}},} \\
& {\left[a_{0}, \cdots, a_{k-1}-1\right]=\frac{p_{k}-p_{k-1}}{q_{k}-q_{k-1}},}
\end{aligned}
$$

from which we see that

$$
\begin{aligned}
& \text { if }\left[a_{0}, \cdots, a_{k-1}\right]=\left[b_{0}, \cdots, b_{l-1}\right]^{-1} \text { and }\left[a_{0}, \cdots, a_{k-1}-1\right] \\
& =\left[b_{0}, \cdots, b_{l-2}\right]^{-1} \text { hold, then }\left[a_{0}, \cdots, a_{k-1}, 2\right] \\
& =\left[b_{0}, \cdots, b_{l-1}+1\right]^{-1} .
\end{aligned}
$$

We shall prove the assertions (i), (ii), and (iii) by induction on the number $\mu=a_{0}+\cdots+a_{k-1}-k$. If $\mu=1$, then $\left(k, a_{0}\right)=(1,2)$ or $\left(k, a_{0}, a_{1}\right)=(2,1,2)$. We then have $[2]=[1,2]^{-1}$ or $[1,2]=[2]^{-1}$, respectively. In these cases the assertions hold trivially. Assume that the assertions are true for the case when $a_{0}+\cdots+a_{k-1}-k$ is at most $\mu(\geqq 1)$, and let $a_{0}+\cdots a_{k-1}-k=\mu+1$ and $\left[a_{0}, \cdots\right.$, $\left.a_{k-1}\right]=\left[b_{0}, \cdots, b_{l-1}\right]^{-1}$. First, assume $a_{k-1} \geqq 3$, and let $\left[c_{0}, \cdots, c_{l^{\prime}-1}\right]$ be the halfregular continued fraction expansion of the number $\left[a_{0}, \cdots, a_{k-1}-1\right]^{-1}$. By the induction hypothesis we see that $c_{0}+\cdots+c_{l^{\prime}-1}-l^{\prime}=\mu$ and $\left[a_{0}, \cdots, a_{k-2}\right]=$ $\left[c_{0}, \cdots, c_{l^{\prime}-1}+1\right]^{-1}$. By (3.8) we see $\left[c_{0}, \cdots, c_{l^{\prime}-1}, 2\right]=\left[a_{0}, \cdots, a_{l_{-1}}\right]^{-1}$. By the uniqueness of the expansion we have $\left(c_{0}, \cdots, c_{l^{\prime}-1}, 2\right)=\left(b_{0}, \cdots, b_{l_{-1}}\right)$, so that $\left[a_{0}, \cdots, a_{k-1}-1\right]=\left[b_{0}, \cdots, b_{l-2}\right]^{-1}$ and $b_{l-1}=2$. Furthermore, $b_{0}+\cdots+b_{l-1}-l=c_{0}$ $+\cdots+c_{l^{\prime}-1}+2-\left(l^{\prime}+1\right)=\mu+1$. Thus, (i), (ii), and (iii) hold. Finally, assume $a_{k-1}=2$, and let $\left[c_{0}, \cdots c_{l^{\prime}-1}\right]$ be the half-regular continued fraction expansion of $\left[a_{0}, \cdots, a_{k-2}\right]^{-1}$. By the induction hypothesis we see that $c_{0}+\cdots+c_{l^{\prime}-1}-l^{\prime}=$ $a_{0}+\cdots+a_{k-2}-(m-1)=\mu,\left[a_{0}, \cdots, a_{k-2}-1\right]=\left[c_{0}, \cdots, c_{l^{\prime}-2}\right]^{-1}$ and $c_{l^{\prime}-2} \geqq 2$ even if $l^{\prime}=1$ because of the fact $\mu \geqq 1$. It follows from (3.8) that $\left[a_{0}, \cdots, a_{k-2}, 2\right]=$ $\left[c_{0}, \cdots, c_{l^{\prime}-1}+1\right]^{-1}$; therefore $\left(c_{0}, \cdots, c_{l^{\prime}-1}+1\right)=\left(b_{0}, \cdots, b_{l-1}\right)$ so that $l^{\prime}=l$, $\left[a_{0}, \cdots, a_{k-2}\right]=\left[b_{0}, \cdots, b_{l-1}-1\right]^{-1}$ and $b_{l-1} \geqq 2$. Let $s=b_{l-1}-2$. By the induction hypothesis we have $a_{j}=2(k-1 \leqq j \leqq k-s)$, and $\left[a_{0}, \cdots, a_{k-s-1}\right]=\left[b_{0}, \cdots, b_{l-2}, 2\right]^{-1}$. Again by the induction hypothesis we get $\left[a_{0}, \cdots, a_{k-s-1}-1\right]=\left[b_{0}, \cdots, b_{l_{-2}}\right]^{-1}$. Since

$$
\begin{aligned}
{\left[a_{0}, \cdots, a_{k-s-1}-1\right] } & =\left[a_{0}, \cdots, a_{k-s-1}, 1\right] \\
& =\left[a_{0}, \cdots, a_{k-s-1}, 2,2, \cdots, 2,1\right] \\
& =\left[a_{0}, \cdots, a_{k-s-1}, a_{k-s}, \cdots, a_{k-2}, a_{k-1}-1\right],
\end{aligned}
$$

we have $\left[a_{0}, \cdots, a_{k-1}-1\right]=\left[b_{0}, \cdots, b_{l-2}\right]^{-1}$. Furthermore, $b_{0}+\cdots+b_{l-1}-l=$ 
$c_{0}+\cdots+\left(c_{l^{\prime}-1}+1\right)-l^{\prime}=\mu+1$. We have obtained all the assertions (i), (ii), (iii) and proved the lemma.

Remark 3.4. Let $\omega=c_{0}+K_{1 \leqq \jmath}\left(1 / c_{\jmath}\right)$ be the regular continued fraction expansion of a real number $\omega$, where $c_{\jmath}$ are integers with $c_{\jmath} \geqq 1(j \geqq 1)$ (cf., e.g., [6], [9]). Let $r_{j} / s$, be the natural representation of the rational number

$$
c_{0}+\frac{1}{c_{1}}+\cdots+\frac{1}{c_{\jmath-1}}=c_{0}+K_{1 \leqq k<j}\left(1 / c_{k}\right)
$$

and set $\gamma_{\jmath}=\left(s_{\jmath}, r_{\jmath}\right)(j \geqq 1), \gamma_{0}=(0,1)$. To get the unique expansion for rational numbers we place the following restriction: the length $M$ of the expansion must be even whenever $M$ is finite. For example, if $\omega=4 / 5$ (resp. 16/9), we expand it as

$$
\frac{4}{5}=0+\frac{1}{1}+\frac{1}{3}+\frac{1}{1} \quad\left(\text { resp. } \frac{16}{9}=1+\frac{1}{1}+\frac{1}{3}+\frac{1}{2}\right)
$$

not

$$
\frac{4}{5}=0+\frac{1}{1}+\frac{1}{4} \quad\left(\text { resp. } \frac{16}{9}=1+\frac{1}{1}+\frac{1}{3}+\frac{1}{1}+\frac{1}{1}\right) .
$$

We then have the following relationship between the regular continued fraction expansion $c_{0}+K_{1 \leqq j<M}\left(1 / c_{j}\right)$ of any non-integral real number $\omega$ and the halfregular continued fraction expansion $a_{0}+K_{1 \leq \jmath<N}\left(-1 / a_{\jmath}\right)$ of $\omega$ with the notation in Lemma 3.1 as well as the convension $a(j)=a_{\jmath}, \alpha(j)=\alpha_{j}$ :

$$
\begin{gathered}
\left\{\begin{array}{l}
c_{0}=a(0)-1, \quad c_{2 \jmath}=a\left(c_{1}+c_{3}+\cdots+c_{2 \jmath-1}\right)-2 \quad(j \geqq 1) \\
c_{2 j+1}=\max \left\{k \geqq 1 ; a\left(c_{1}+c_{3}+\cdots+c_{2 \jmath-1}+k\right) \neq 2\right\} \quad(j \geqq 0),
\end{array}\right. \\
\left\{\begin{array}{l}
\gamma_{0}=\alpha(0), \quad \gamma_{2 \jmath}=\alpha\left(c_{1}+c_{3}+\cdots+c_{2 \jmath-1}\right) \quad(j \geqq 1) \\
\gamma_{2 j+1}=\alpha\left(c_{1}+c_{3}+\cdots+c_{2 j-1}+k\right)-\alpha\left(c_{1}+c_{3}+\cdots+c_{2 \jmath-1}+k-1\right)
\end{array}\right. \\
\left(j \geqq 0,1 \leqq k \leqq c_{2 j+1}\right) .
\end{gathered}
$$

Conversely,

$$
\begin{aligned}
& \left\{\begin{array}{l}
a(0)=c_{0}+1, \quad a\left(c_{1}+c_{3}+\cdots+c_{2 j-1}\right)=c_{2 j}+2 \quad(j \geqq 1) \\
a\left(c_{1}+c_{3}+\cdots+c_{2 \jmath-1}+k\right)=2 \quad\left(1 \leqq k \leqq c_{2 j+1}-1, j \geqq 0\right),
\end{array}\right. \\
& \alpha\left(c_{1}+c_{3}+\cdots+c_{2 \jmath-1}+k\right)=\gamma_{2 j}+k \gamma_{2 j+1} \quad\left(0 \leqq k \leqq c_{2 j+1}-1, j \geqq 0\right) .
\end{aligned}
$$

In terms of the regular continued fraction expansion of $\omega$, the assertions in Remark 3.2 and Lemma 3.1 are written as follows:

$$
\begin{aligned}
S \backslash(S+S)= & \left\{\gamma_{0}\right\} \cup\left\{\gamma_{2 j}+k \gamma_{2 j+1} ; 0 \leqq j<M / 2,1 \leqq k \leqq c_{2 j+1}\right\}, \\
& \text { Vert } S=\left\{\gamma_{2 j} ; 0 \leqq j<M / 2+1\right\} .
\end{aligned}
$$




\section{Two dimensional case.}

Let $D$ be a logarithmically coned, complete Reinhardt domain in $C^{2}$. Assume $D \neq C^{2}$. There then exist real numbers $\gamma, \delta$ with $(1 / 2) \pi \leqq \gamma \leqq \pi,(3 / 2) \pi \leqq \delta \leqq 2 \pi$ such that

$$
\log |D|=\left\{(r \cos \theta, r \sin \theta) \in \boldsymbol{R}^{2} ; r>0, r<\theta<\delta\right\} .
$$

If $\delta>\gamma+\pi$, then

$$
D=\left\{z \in \boldsymbol{C}^{2} ;\left|z_{1}\right|^{-\tan \gamma}\left|z_{2}\right|<1\right\} \cup\left\{z \in \boldsymbol{C}^{2} ;\left|z_{1}\right|^{-\tan \delta}\left|z_{2}\right|<1\right\},
$$

and $S_{D}=\phi$, so that $c_{D}^{*}(0, z)=0$. Since $D$ is not pseudoconvex, by Lemma 1.1 we see that $\exp g_{D}(0, z)<\mu_{D}(z)$ for some $z \in D$, with

$$
\mu_{D}(z)=\min \left\{\left(\left|z_{1}\right|^{-\tan \gamma}\left|z_{2}\right|\right)^{1 /(1-\tan \gamma)}, \quad\left(\left|z_{1}\right|^{-\tan \delta}\left|z_{2}\right|\right)^{1 /(1-\tan \delta)}\right\} .
$$

Next, assume $\delta=\gamma+\pi$. Then, $D=\left\{z \in \boldsymbol{C}^{2} ;\left|z_{1}\right|^{-\tan \gamma}\left|z_{2}\right|<1\right\}$, and

$$
\exp g_{D}(0, z)=k_{D}^{*}(0, z)=\mu_{D}(z)=\left(\left|z_{1}\right|^{-\tan \gamma}\left|z_{2}\right|\right)^{1 /(1-\tan \gamma)} .
$$

If $\tan \gamma$ is an irrational number, then $S_{D}=\phi$ and $c_{D}^{*}(0, z)=0$; while if $\tan \gamma$ is a rational number $-q / p$ with natural representation, then $S_{D}=\left(\boldsymbol{Z}_{+}\right)_{*}(q, p)$, and $S_{D} \backslash\left(S_{D}+S_{D}\right)=\operatorname{Vert} S_{D}=\{(q, p)\}$, so that $c_{D}^{*}(0, z)=\left|z_{1}\right|^{q}\left|z_{2}\right|^{p}$ for $z \in D$, while $k_{D}^{*}(0, z)=\left(\left|z_{1}\right|^{q}\left|z_{2}\right|^{p}\right)^{1 /(q+p)}$.

Finally we assume that $\delta<\gamma+\pi$. Then,

$$
D=\left\{z \in C^{2} ;\left|z_{1}\right|^{-\tan \gamma}\left|z_{2}\right|<1,\left|z_{1}\right|^{-\tan \delta}\left|z_{2}\right|<1\right\} .
$$

Setting $\tau=-\tan \gamma, \omega=-1 / \tan \delta$, we have

$$
0 \leqq \omega<1 / \tau \leqq+\infty,
$$

and

$$
D=\left\{z \in C^{2} ;\left|z_{1}\right|\left|z_{2}\right|^{\omega}<1,\left|z_{1}\right|^{\tau}\left|z_{2}\right|<1\right\} .
$$

Setting

$$
\alpha_{\infty}=(1, \omega), \quad \beta_{\infty}=(\tau, 1),
$$

we have

$$
S_{D}=\left(\boldsymbol{Z}^{2}\right)_{*} \cap\left(\boldsymbol{R}_{+} \alpha_{\infty}+\boldsymbol{R}_{+} \beta^{\infty}\right) .
$$

By Lemma 1.1 (iii) we have 


$$
\begin{aligned}
\exp g_{D}(0, z) & =k_{D}^{*}(0, z)=\mu_{D}(z) \\
& =\max \left\{\left(\left|z_{1}\right|\left|z_{2}\right|^{\omega}\right)^{1 /(1+\omega)},\left(\left|z_{1}\right|^{\tau}\left|z_{2}\right|\right)^{1 /(\tau+1)}\right\} .
\end{aligned}
$$

For the Carathéodory pseudodistance we have the following.

THEOREM 4.1. Let $\omega$ and $\tau$ be two real numbers satisfying (4.1), and let $\omega=a_{0}+K_{1 \leqq m<M}\left(-1 / a_{m}\right), \tau=b_{0}+K_{1 \leqq n<N}\left(-1 / b_{n}\right)$ be the half-regular continued fraction expansions. Let $p_{m} / q_{m}=\left[a_{0}, \cdots, a_{m-1}\right], s_{n} / r_{n}=\left[b_{0}, \cdots, b_{n-1}\right]$ be the natural representations, and set $\alpha_{m}=\left(q_{m}, p_{m}\right), \beta_{n}=\left(s_{n}, r_{n}\right)$ with $\alpha_{0}=(0,1), \beta_{0}=(1,0)$.

(i) There exist unique integers $m_{0} \geqq 0, n_{0} \geqq 0$ such that $\alpha_{m_{0}}=\beta_{n_{0}}=: \gamma$.

(ii) If $D$ is the domain defined by (4.2), and if $E_{D}=\{\gamma\} \cup\left\{\alpha_{m} ; m_{0}<m<M+1\right.$, $\left.a_{m} \neq 2\right\} \cup\left\{\beta_{n} ; n_{0}<n<N+1, b_{n} \neq 2\right\}$, then Vert $S_{D}=E_{D}$ (see (3.7)); therefore $c_{D}^{*}(0, z)=\max \left\{\left|z^{\alpha}\right| ; \alpha \in E_{D}\right\}$ for all $z \in D$.

Proof. To prove (i), assume $1 / \tau=+\infty$, or $\tau=0$. Then, $b_{0}=0$, and $\beta_{1}=(0,1)$, so that $\alpha_{0}=\beta_{1}$. Next, assume $0 \leqq \omega<1 / \tau<+\infty$. Since the sequence $\left(\left[a_{0}, \cdots, a_{m-1}\right]\right)_{m}$ is strictly decreasing and converges to $\omega$, there exists an integer $m_{0} \geqq 0$ such that $\left[a_{0}, \cdots, a_{m_{0}}\right]<1 / \tau \leqq\left[a_{0}, \cdots, a_{m_{0^{-1}}}\right]$, or $\left[a_{0}, \cdots, a_{m_{0^{-1}}}\right]^{-1} \leqq \tau<\left[a_{0}, \cdots, a_{m_{0}}\right]^{-1}$. Let $\left[c_{0}, \cdots, c_{\jmath_{-1}}\right]$ be the half-regular continued fraction expansion of the number $\left[a_{0}, \cdots, a_{m_{0}}\right]^{-1}$. Then, by Lemma 3.2 we have $\left[c_{0}, \cdots, c_{\jmath-1}-1\right]=\left[a_{0}, \cdots, a_{m_{0}-1}\right]^{-1}$. If $\left[a_{0}, \cdots, a_{m_{0-1}}\right]^{-1}=\tau$, then $\tau=\left[c_{0}, \cdots, c_{\jmath_{-1}}-1\right]=\left[c_{0}, \cdots, c_{n_{0-1}}-1\right]$, where $n_{0}=$ $\max \Delta$ if $\Delta:=\left\{l \in\{1, \cdots, j\} ; c_{l-1} \neq 2\right\} \neq \phi$ and $n_{0}=1$ if $\Delta=\phi$. Thus, $\left[b_{0}, \cdots, b_{N-1}\right]$ $=\left[c_{0}, \cdots, c_{n_{0^{-1}}}-1\right]$ and $n_{0}=N$; therefore $\alpha_{m_{0}}=\beta_{N}$. Next, assume $\left[a_{0}, \cdots, a_{m_{0-1}}\right]^{-1}$ $<\tau$. Since the interval $\left[c_{0}, \cdots, c_{\jmath-1}-1\right]<t<\left[c_{0}, \cdots, c_{\jmath-1}\right]$ coincides with $\left\{\left[c^{0}, \cdots\right.\right.$, $\left.\left.c_{\jmath-1}, w\right] ; w>1\right\}$, there exists a real number $w>1$ such that $\tau=\left[c_{0}, \cdots, c_{\jmath-1}, w\right]$. Since $\tau=\left[b_{0}, \cdots, b_{j-1}, \omega_{j}\right]$ for some $\omega_{j}>1$, we see that $\left(c_{0}, \cdots, c_{\jmath-1}\right)=\left(b_{0}, \cdots, b_{\jmath-1}\right)$, so that $\left[a_{0}, \cdots, a_{m_{0}}\right]^{-1}=\left[b_{0}, \cdots, b_{j-1}\right]$, or $\alpha_{m_{0}}=\beta_{j}$. We thus proved the assertion (i).

To prove (ii), let $\alpha_{\infty}, \beta_{\infty}$ be as in (4.3), and let $S_{1}=\left(\boldsymbol{Z}^{2}\right)_{*} \cap\left(\boldsymbol{R}_{+} \alpha_{0}+\boldsymbol{R}_{+} \alpha_{\infty}\right)$, $S_{2}=\left(\boldsymbol{Z}^{2}\right)_{*} \cap\left(\boldsymbol{R}_{+} \beta_{0}+\boldsymbol{R}_{+} \beta_{\infty}\right)$. It follows from (4.4) that $S_{D}=S_{1} \cap S_{2}$. By Lemma 3.1 we see that

$$
\begin{aligned}
& \left\{\alpha_{m} ; m_{0}<m<M+1, a_{m} \neq 2\right\} \subset\left(\operatorname{Vert} S_{1}\right) \cap S_{2} \subset \operatorname{Vert} S_{D}, \\
& \left\{\beta_{n} ; n_{0}<n<N+1, b_{n} \neq 2\right\} \subset\left(\operatorname{Vert} S_{2}\right) \cap S_{1} \subset \operatorname{Ver} t S_{D} .
\end{aligned}
$$

Furthermore, since $\operatorname{grad}\left(\gamma, \alpha_{m_{0}+1}\right) \leqq \omega<1 / \tau \leqq \operatorname{grad}\left(\gamma, \beta_{n_{0}+1}\right)$, we see that $\gamma \in \operatorname{Vert} S_{D}$. Thus, $E_{D} \subset$ Vert $S_{D}$. Conversely, let $\alpha \in \operatorname{Vert} S_{D}$ and $\alpha \neq \gamma$. Then $\operatorname{grad}(0, \alpha) \neq$ $\operatorname{grad}(0, \gamma)$. Assume $\operatorname{grad}(0, \alpha)<\operatorname{grad}(0, \gamma)$. There then exists a linear functional $f$ on $\boldsymbol{R}^{2}$ and $c \in \boldsymbol{R}$ such that $f(\alpha)=c, f(x)<c$ for all $x \in S_{D} \backslash\{\alpha\}$. Let $g$ be the gradient of the line $f^{-1}(c)$. Then, $\operatorname{grad}\left(\gamma, \alpha_{m_{0}+1}\right) \leqq \operatorname{grad}(\gamma, \alpha) \leqq g \leqq$ $\operatorname{grad}\left(0, \alpha_{\infty}\right) \leqq \operatorname{grad}(0, \gamma)$. It follows that the set $S_{1} \backslash S_{2}$ is contained in the cone $\gamma+\boldsymbol{R}_{+}\left(\gamma-\alpha_{m_{0}+1}\right)+\boldsymbol{R}_{+} \gamma$, and that $f(x)<c$ for all $x \in S_{1} \backslash\{\alpha\}$; therefore, $\alpha \in \operatorname{Vert} S_{1}$, and $\alpha=\alpha_{m}$ for some $m$ with $m_{0}<m<M+1$ and $a_{m} \neq 2$ (by Lemma 3.1). Similarly, if $\operatorname{grad}(0, \alpha)>\operatorname{grad}(0, \gamma)$, then $\alpha \in \operatorname{Vert} S_{2}$, and $\alpha=\beta_{n}$ for some $n$ with 
$n_{0}<n<N+1$ and $b_{n} \neq 2$. We thus have proved Vert $S_{D} \subset E_{D}$ and the theorem.

Remark 4.2. Let $\omega, \tau, D$, and $E_{D}$ be as in Theorem 4.1. Let $\omega=$ $a_{0}+K_{1 \leqq m<M}\left(1 / a_{m}\right)$ and $\tau=b_{0}+K_{1 \leqq n<N}\left(1 / b_{n}\right)$ be the regular continued fraction expansions of $\omega$ and $\tau$ (see Remark 3.4). Represent $p_{m} / q_{m}=a_{0}+K_{1 \leqq \jmath<m}\left(1 / a_{\jmath}\right)$ and $s_{n} / r_{n}=b_{0}+K_{1 \leq \jmath<n}\left(1 / b_{j}\right)$ naturally, and set $\alpha_{m}=\left(q_{m}, p_{m}\right), \beta_{n}=\left(s_{n}, r_{n}\right)$ with $\alpha_{0}=(0,1), \beta_{0}=(1,0)$. There then exist non-negative integers $m_{0}, n_{0}, j_{0}$, and $k_{0}$ such that $\alpha_{2 m_{0}}+j_{0} \alpha_{2 m_{0}+1}=\beta_{2 n_{0}}+k_{0} \beta_{2 n_{0}+1}:=\gamma$, and it holds that $E_{D}=\{\gamma\} \cup\left\{\alpha_{2 m}\right.$; $\left.m_{0}<m<M / 2+1\right\} \cup\left\{\beta_{2 n} ; n_{0}<n<N / 2+1\right\}$.

Finally we present some examples which are applicable to Theorem 4.1.

Example 1 ([7], [8]). Let $D=\left\{z \in C^{2} ;\left|z_{1}\right|<1,\left|z_{1}\right|^{345}\left|z_{2}\right|^{128}<1\right\}$. In this case we have $\omega=0, \tau=345 / 128=[3,4,2,2,3,6]$, and the following table:

\begin{tabular}{l|rrrrrrr}
$b_{n}$ & 3 & 4 & 2 & 2 & 3 & 6 & $+\infty$ \\
\hline$s_{n}$ & 1 & 3 & 11 & 19 & 27 & 62 & 345 \\
$r_{n}$ & 0 & 1 & 4 & 7 & 10 & 23 & 128
\end{tabular}

It follows that $E_{D}=\{(1,0),(3,1),(27,10),(62,23),(345,128)\}$. Thus $c_{D}^{*}(0, z)=$ $\max \left\{\left|z^{\alpha}\right| ; \alpha \in E_{D}\right\}$.

Example 2. Let $D=\left\{z \in C^{2} ;\left|z_{1}\right|^{2}\left|z_{2}\right|^{7}<1,\left|z_{1}\right|^{\sqrt{2}}\left|z_{2}\right|<1\right\}$. Noting $0<1 / \sqrt{2}<$ $7 / 2$, we have $\omega=1 / \sqrt{2}=[1,4,2,4,2,4, \cdots], \tau=2 / 7=[1,2,2,3]$, and the tables :

\begin{tabular}{|c|c|c|c|c|c|c|c|c|c|c|c|c|c|}
\hline$a_{m}$ & 1 & 4 & 2 & 4 & 2 & 4 & $\ldots$ & $b_{n}$ & 1 & 2 & 2 & 3 & $+\infty$ \\
\hline$q_{m}$ & 0 & 1 & 4 & 7 & 24 & 41 & $\ldots$ & $s_{n}$ & 1 & 1 & 1 & 1 & 2 \\
\hline$p_{m}$ & 1 & 1 & 3 & 5 & 17 & 29 & $\ldots$ & $r_{n}$ & 0 & 1 & 2 & 3 & 7 \\
\hline
\end{tabular}

It follows that $\alpha_{1}=\beta_{1}$, and $E_{D}=\{(1,1)\} \cup\left\{\alpha_{2 m+1} ; m \geqq 1\right\} \cup\{(1,3),(2,7)\}$, where $\alpha_{2 m+1}=6 \alpha_{2 m-1}-\alpha_{2 m-3} \quad(m \geqq 2), \quad \alpha_{1}=(1,1), \quad \alpha_{3}=(7,5)$. Thus $c_{D}^{*}(0, z)=\max \left\{\left|z^{\alpha}\right|\right.$ : $\left.\alpha \in E_{D}\right\}$.

\section{REFERENCES}

[1] K. Azukawa, Two intrinsic pseudometrics with pseudoconvex indicatrices and starlike circular domains, J. Math. Soc. Japan, 38 (1986), 627-647.

[2] K. Azukawa, The invariant pseudo-metric related to negative pluri-subharmonic functions, Kodai Math. J., 10 (1987), 83-92.

[3] E. BedFord ANd J.-P. Demailly, Two counterexamples concerning the pluricomplex Green function in $\boldsymbol{C}^{n}$, Indiana Univ. Math. J., 37 (1988), 865-867.

[4] J.-P. Demailly, Mesures de Monge-Ampère et mesures pluriharmoniques, Math. Z., 194 (1987), 519-564. 
[5] S. Dineen, The Schwarz Lemma, Oxford Univ. Press, Oxford, 1989.

[6] M.C. IRwin, Geometry of continued fractions, Amer. Math. Monthly, 96 (1989), 696-703.

[7] M. Jarnicki and P. Pflug, Three remarks about the Caratheodory distance, Deformations of Mathematical Structures, J. Ławrynowicz (ed.), Kluwer Academic Publishers, Dordrecht, 1989, pp. 161-170.

[8] M. Jarnicki And P. Pflug, Effective formulas for the Carathéodory distance, Manuscripta Math., 62 (1988), 1-22.

[9] W.B. Jones AND W. J. Thron, Continued Fraction-Analytic Theory and Applications, Encyclopedia of Mathematics and Its Applications 11, Addison-Wesley, London, 1980.

[10] M. KLIMEK, Extremal plurisubharmonic functions and invariant pseudodistances, Bull. Soc. Math. France, 113 (1985), 231-240.

[11] S. Kobayashi, Hyperbolic Manifolds and Holomorphic Mappings, Marcel Dekker, New York, 1970.

[12] A. Kodama, A simple proof of Kubota's theorem on the Kobayashi and Carathéodory distances, Math. Rep. Toyama Univ., 12 (1989), 201-205.

[13] Y. Kubota, On the Kobayashi and Carathéodory distances of bounded symmetric domains, Kodai Math. J., 12 (1989), 41-48.

[14] L. LEMPERT, La métrique de Kobayashi et la représentation des domaines sur la boule, Bull. Soc. Math. France, 109 (1981), 427-474.

[15] E. A. PoletskiI And B. V. Shabat, Invariant metrics, Encyclopedia of Mathematical Sciences 9, Several Complex Variavles III, G. M. Khenkin (ed.), Springer, Berlin, 1989, pp. 63-111.

[16] O. RIEMENSCHNEIDER, Deformationen von Quotientensingularitäten (nach zyklischen Gruppen), Math. Ann., 209 (1974), 211-248.

\author{
Department of Mathematics \\ FACULTY OF SCIENCE \\ TOYAMA UNIVERSITY \\ Gofuku, TOYAMa 930, JAPAN
}

